\title{
TOPOLOGICAL EQUIVALENCE OF GRADIENT VECTORFIELDS
} BY

\author{
DOUGLAS S. SHAFER
}

\begin{abstract}
This paper is a study of the behavior of the topological equivalence class of the planar gradient vectorfield $X=\operatorname{grad}_{g} V$, in a neighborhood of a degenerate singularity of $V$, as $g$ varies over all Riemannian metrics. It is shown that under simple restrictions on $V$ the topological equivalence class of $X$ is determined by its first nonvanishing jet, and that only finitely many equivalence classes occur (for fixed $V$ ). In this case, when the degree of the first nonvanishing jet of $V$ is less than five, necessary and sufficient conditions for change in equivalence class are given, both in terms of the coefficients of the homogeneous part of $V$ and geometrically in terms of its level curves. A catalogue of possible phase portraits, up to topological equivalence, is included. Necessary conditions are given for change in higher degree.
\end{abstract}

0. Introduction. In [5, p. 229] René Thom posed the following problem: "Find an example of a polynomial $P: R^{n} \rightarrow R$ such that by changing the ambient Riemannian metric the topological type of the set of gradient trajectories of $P$ arriving at 0 may vary." Floris Takens produced $P(x, y)=x\left(2 x^{2}+3 y^{2}\right)$ as such an example [5, p. 231]. A more general problem, mentioned by John Guckenheimer in [3, p. 105], is that of determining the extend to which, for a fixed potential function $V$, the topological equivalence class of $\operatorname{grad}_{g} V$ can vary as the Riemannian metric $g$ is varied. This paper gives a fairly complete answer to the latter problem in the plane, with general results as described in the abstract.

\section{Preliminaries.}

(1.1) Definition. Let $X$ and $Y$ be at least $C^{1}$ vectorfields defined on a nbd of 0 in $R^{n}$, with critical points at 0 . Then $X$ and $Y$ are topologically equivalent (i.e., their germs are equivalent), and are said to have the same topological type, in a nbd of 0 if there exists a homeomorphism of a nbd of $\mathbf{0}$ onto its image carrying oriented orbits of $X$ onto oriented orbits of $Y$.

A similar but stronger notion is that of topological conjugacy, in which the equivalence homeomorphism must preserve not just the sense of the orbits but their parametrization.

Let $M$ be a smooth finite-dimensional manifold and $V: M \rightarrow R$ be $C^{k}, k>2$. If $V$ has a singularity at a point $p$ in $M$, then for any Riemannian metric $g$ the gradient vectorfield $X=\operatorname{grad}_{g} V$ has a stationary point at $p$, and it makes sense to ask how the structure of $X$ near $p$ depends on $g$. Specifically, how does the topological equivalence class of $X$ vary as the Riemannian metric $g$ varies? Since

Received by the editors September 19, 1978 and, in revised form, February 21, 1979.

AMS (MOS) subject classifications (1970). Primary 34C05, 34C35, 34D30; Secondary 58F99.

Key words and phrases. Gradient vectorfield, topological equivalence. 
the question is a purely local one we will henceforth assume that $M$ is a nbd of 0 in $R^{n}$, that $p=0$, and that $V(0)=0$.

It is well known that when the singularity at 0 is nondegenerate the topological type of $X=\operatorname{grad}_{g} V$ is independent of $g$, and that in fact vectorfields arising from different Riemannian metrics are not only equivalent but conjugate in a nbd of 0 . The same result holds when the singularity of $V$ at 0 is degenerate but 0 is an attractor (or repeller) for $X$, i.e., is the alpha (resp. omega) limit set for every point in a nbd of 0 . For then 0 is an isolated relative maximum (resp. minimum) for $V$, there is a nbd of 0 that is positively (resp. negatively) invariant for $X$, for every $g$, and a conjugacy can be constructed by standard "first intersection" techniques.

This paper then deals with an isolated degenerate singularity at 0 which is not an attractor for either $X$ or $-X$, with $n=2$. Since the first partials of $V$ vanish simultaneously at $(0,0)$, but nowhere else, the following proposition, which is in fact an exercise in Hartman [4, p. 173], describes the structure of the level curves of $V$.

(1.2) Proposition [4]. Let $V$ be $C^{k}, k \geqslant 1$, satisfying $V_{x}(p)=V_{y}(p)=0$ iff $p=(0,0)$. Then either (i) there exists an $\varepsilon>0$ such that $0<\|p\|<\varepsilon$ implies $V(p) \neq 0$, in which case $V$ has a strict maximum or minimum at $(0,0)$ and for $k$ near 0 (below or above, respectively) $V^{-1}(k)$ is a topological circle about $(0,0)$; or (ii) the set of arcs which join $(0,0)$ and $\{p:\|p\|=\varepsilon\}$, some $\varepsilon>0$, and on which $V=0$, consists of a finite even number $2 m$ of arcs, $2 m>0$.

It is clear that in case (i) the critical point of $X$ (or of $-X$ ) at $(0,0)$ is asymptotically stable. The following proposition describes the structure of $X$ near $(0,0)$ when case (ii) holds.

(1.3) Proposition. If $V$ is as in Proposition 1.2 and case (ii) holds, then for every Riemannian metric $g$ there is a one-to-one correspondence between hyperbolic sectors of $X=\operatorname{grad}_{g} V$ and the arcs of which $V^{-1}(0)$ consists.

(1.4) COROLlaRY. The number of hyperbolic sectors of $X=\operatorname{grad}_{g} V$ is independent of the Riemannian metric $g$, as is the index of $X$ at $(0,0)$.

Proof of (1.3). We sketch the proof, using the terminology of [4]. For $\varepsilon$ as in Proposition 1.2, case (ii), if $0<\|p\|<\varepsilon$ and $V(p)=0$, then the trajectory through $p$ leaves $\{x:\|x\| \leqslant \varepsilon\}$ in forward and reverse time, since it cannot tend to $(0,0)$. Thus $p$ and the points on the arc in $V^{-1}(0)$ connecting it to $(0,0)$ are in the hyperbolic portion of the sector $S$ containing $p$; but then so is $(0,0)$, so $S$ is hyperbolic. If $p$ and $q$ are on the same arc of $V^{-1}(0)$ they are clearly in the same hyperbolic sector. If they are in different arcs, consideration of the trajectories in forward and reverse time of a sequence of points $\left(x_{i}\right)$ in the same arc of $V^{-1}(0)$ as $p$ and tending to $(0,0)$ leads to the existence of base solutions separating $p$ and $q$. Finally, if $S$ is a hyperbolic sector, consideration of the trajectories of the associated Hamiltonian vectorfield crossing the positive and negative base solutions of $S$ yields an arc of zeros of $V$ in $S$. 
Since the number of hyperbolic sectors of $X=\operatorname{grad}_{g} V$ is fixed independently of the Riemannian metric, and since as a gradient $X$ has no elliptic sectors, the change in structure of $X$ near $(0,0)$ with change in $g$ is limited to possible "opening" or "closing" of parabolic sectors. This leads to the following result.

(1.5) THEOREM. If $V$ has an isolated singularity at $(0,0)$ then the number of distinct topological equivalence classes of $X=\operatorname{grad}_{g} V$, as $g$ varies over all Riemannian metrics, is finite and less than $2^{h}$, where $h$ is the number of hyperbolic sectors of $X$ at $(0,0)$, independent of $g$.

Proof. If there are $h$ hyperbolic sectors then between any two of them there either is or is not a parabolic sector. Thus there are at most $2^{h}$ possible configurations of hyperbolic and parabolic sectors of $X$ at $(0,0)$. The theorem is now a consequence of the following lemma, a special case of a more general result proved in [1, Chapter VIII].

(1.6) LEMMA [1]. If vectorfields $X$ and $Y$ have no elliptic sectors and the same configuration of a finite number of hyperbolic and parabolic sectors at $(0,0)$, then they are topologically equivalent in a nbd of $(0,0)$.

2. Determinacy by a finite jet. We now know that the number of distinct topological equivalence classes of $X=\operatorname{grad}_{g} V$ near an isolated singular point of $V$ in $R^{2}$ remains finite as $g$ is varied, and that change is confined to appearance or disappearance of parabolic sectors. If the singularity of $V$ at $(0,0)$ were nondegenerate then the critical point of $X$ at $(0,0)$ would be hyperbolic and by the Hartman-Grobman Theorem determined by the linear part of $X$ at $(0,0)$. In this section the blowing-up construction of F. Takens is introduced and is used to obtain conditions under which, at a degenerate singularity of $V, X$ is still determined by a finite jet. The construction is immediately applied to the case of potentials which vanish to first order at $(0,0)$, and phase portraits are obtained.

For $X$ a $C^{r}$ vectorfield on $R^{2}$, let

$$
\alpha_{r}=\left\langle x^{1} \partial / \partial x^{1}+x^{2} \partial / \partial x^{2}, X\right\rangle: R^{2} \rightarrow R
$$

and

$$
\alpha_{t}=\left\langle x^{1} \partial / \partial x^{2}-x^{2} \partial / \partial x^{1}, X\right\rangle: R^{2} \rightarrow R
$$

where $\langle$,$\rangle is the usual inner product on R^{2}$.

(2.1) Proposition [7]. Let $X$ be a $C^{r}$ vectorfield on a nbd of $(0,0)$ in $R^{2}$ that vanishes at $(0,0)$. Let $\Phi: S^{1} \times R \rightarrow R^{2}:(\phi, r) \mapsto(r \cos \phi, r \sin \phi)$. Then the vectorfield

$$
\tilde{X}(\phi, r)=r^{-2}\left\{\alpha_{r} \circ \Phi(\phi, r) r \partial / \partial r+\alpha_{t} \circ \Phi(\phi, r) \partial / \partial \phi\right\}
$$

on $S^{1} \times R$, defined at $r=0$ by the limit, is $C^{r-1}$ and satisfies $d \Phi_{p}(\tilde{X}(p))=X(p)$ for every $p \in S^{1} \times R$.

If $X$ has $(k-1)$-jet at $(0,0)$ zero, $k \geqslant 2$, then $\tilde{X}$ vanishes on $r=0$. Since this set has codimension 1 , it is possible to divide $\tilde{X}$ successively by $r$, losing one degree of differentiability each time. Then $\bar{X}=r^{-(k-1)} \tilde{X}$ is $C^{r-k}, \Phi_{*} \bar{X}=\Phi_{*} \tilde{X}=X$, and $\bar{X}$ 
is not identically zero on $r=0$. In such cases we require $r-k \geqslant 1$, i.e., $r>k+1$. Then the structure of $\bar{X}$ near $S^{1} \times\{0\}=\Phi^{-1}(0)$ reveals that of $X$ near 0 .

Henceforth we consider potentials of the form $V=U+W$, where, in the usual coordinates $(x, y)$ on $R^{2}, U$ is a homogeneous polynomial of degree $k$ in $x$ and $y$ and $W$ is an at least $C^{k+2}$ function which vanishes together with all its partials up to order $k$ at $(0,0)$. Then $X=\operatorname{grad}_{g} V$ may be written

$$
X(x, y)=\left(\begin{array}{ll}
a(x, y) & b(x, y) \\
b(x, y) & c(c, y)
\end{array}\right)\left(\begin{array}{l}
V_{x}(x, y) \\
V_{y}(x, y)
\end{array}\right)
$$

or

$$
\begin{aligned}
X(x, y)= & \left(a(x, y) V_{x}(x, y)+b(x, y) V_{y}(x, y)\right) \partial / \partial x \\
& +\left(b(x, y) V_{x}(x, y)+c(x, y) V_{y}(x, y)\right) \partial / \partial y
\end{aligned}
$$

where $\left(\begin{array}{ll}a & b \\ b & c\end{array}\right)(x, y)$ is the inverse of the matrix of $g$ in these coordinates and is positive definite for all $(x, y)$.

Before stating the main theorem on determinacy by a finite jet, we use the blowing-up construction to treat the case $\operatorname{deg}(U)=2$.

(2.2) Theorem. Let $V(x, y)=A x^{2}+B x y+C y^{2}+W(x, y)$, where $W$ is at least $C^{4}$ and vanishes together with all its partials through order 2 at $(0,0)$. If $V_{x}$ and $V_{y}$ vanish together only at $(0,0)$, then the topological type of $X=\operatorname{grad}_{g} V$ is independent of the Riemannian metric $g$.

Proof. The linear part of $X$ at $(0,0)$ has eigenvalues

$$
\lambda=(A a+B b+C c) \pm\left[(A a+B b+C c)^{2}-\left(a c-b^{2}\right)\left(4 A C-B^{2}\right)\right]^{1 / 2},
$$

where $a=a(0,0)$, etc. By positive definiteness of the matrix in (1) the radicand is nonnegative. Hence if $4 A C-B^{2} \neq 0$ the critical point is nondegenerate and the result follows.

If $4 A C-B^{2}=0$ then the eigenvalues are 0 and $2(A a+B b+C c)$, and again $a c-b^{2}>0$ implies that the second is nonzero. By the equation satisfied by $A, B$, and $C$ there is a rotation of the coordinate axes such that in the new coordinates $U$ is of the form $U(x, y)=D y^{2}, D \neq 0$, and $X(x, y)=(2 D b y+\cdots) \partial / \partial x+(2 D c y$ $+\cdots) \partial / \partial y$. By definition

$$
\begin{aligned}
& \alpha_{t}=x \partial / \partial y-y \partial / \partial x, \quad X(x, y)=2 D c x y-2 D b y^{2}+\cdots \\
& \alpha_{r}=x \partial / \partial x+y \partial / \partial y, \quad X(x, y)=2 D b x y+2 D c y^{2}+\cdots .
\end{aligned}
$$

Using the coordinates $(\phi, r)$ on $S^{1} \times R$ the blow-up is

$$
\begin{aligned}
\bar{X}(\phi, r)=\tilde{X}(\phi, r)= & {[2 D \sin \phi(c \cos \phi-b \sin \phi)+\cdots] \partial / \partial \phi } \\
& +[2 D \sin \phi(b \cos \phi+c \sin \phi)+\cdots] r \partial / \partial r .
\end{aligned}
$$

The lowest order terms determine the critical points of $\bar{X} \mid S^{1} \times\{0\}$; a pair occurs at $\phi=0, \pi$ and a second pair occurs at $\phi=\phi_{0}, \phi_{0}+\pi$, some $\phi_{0} \neq 0$. The second pair is in fact hyperbolic saddles. For the first pair we expand $\bar{X}$ near $\phi=0$ using $\sin \phi=\phi-(1 / 6) \phi^{3}+\cdots$ and $\cos \phi=1+\frac{1}{2} \phi^{2}-\cdots$, obtaining 


$$
\begin{aligned}
\bar{X}(\phi, r)= & \left(2 D c \phi-2 D b \phi^{2}+A_{1} r+A_{2} r \phi+\cdots\right) \partial / \partial \phi \\
& +\left(2 D b \phi+2 D c \phi^{2}+A_{3} r+A_{4} r \phi+\cdots\right) r \partial / \partial r \quad(\text { near } \phi=0)
\end{aligned}
$$

where the $A_{i}$ depend on $a, b, c$, and coefficients of third order terms in $V$. Since the linear part of $\bar{X}$ at $(\phi, r)=(0,0)$ is $\left(\begin{array}{ll}2 D c \\ 0\end{array}\right)$, with eigenvalues $2 D c \neq 0$ and 0 , any centermanifold $W^{c}$ at $(\phi, r)=(0,0)$ is normally hyperbolic. Because $X$ has no singularities other than the origin in a nbd of the origin, $\bar{X}$ has no singularities off $S^{1} \times\{0\}$, and $\bar{X} \mid W^{c}$ is either expanding or contracting.

Assume $D>0$ (the case $D<0$ is the same). Then $\bar{X} \mid S^{1} \times\{0\}$ has a hyperbolic sink at $\phi=0$. When $\bar{X}$ expands $W^{c}$ radially outward, $W^{c}$ is in fact the unique fixed point of the graph transform that defines it, and, near $\phi=0, \bar{X}$ is equivalent to $-x \partial / \partial x+y \partial / \partial y$ on $y \geqslant 0$. If $\bar{X}$ contracts $W^{c}, \phi=0$ is a sink for $\bar{X}$ in $S^{1} \times[0, \varepsilon)$, $\varepsilon>0$ small. Applying a similar argument at $\phi=\pi$, the structure of $\bar{X}$ in $S^{1} \times$ $[0, \varepsilon)$ is completely determined as one of the four possibilities shown in Figures la-1d (shown for $D>0$ ), according as (depending on higher terms) the flow along the centermanifolds at $(\phi, r)=(0,0)$ and $(0, \pi)$ is, respectively, in-in, out-in, in-out, or out-out. Blowing-down by means of $\Phi$, the corresponding phase portraits, up to topological equivalence, are obtained for $X$ near $(0,0)$ (Lemma 1.6). Then distinct topological equivalence classes of $X$ have different numbers of hyperbolic sectors; hence each must be invariant under changes in the Riemannian metric.

1a.
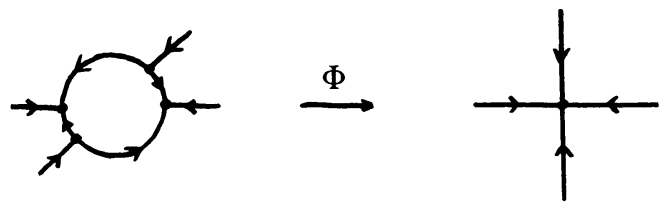

$1 \mathrm{~b}$.
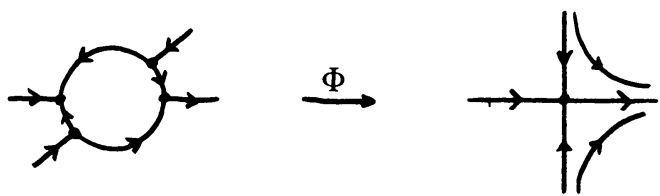

1c.
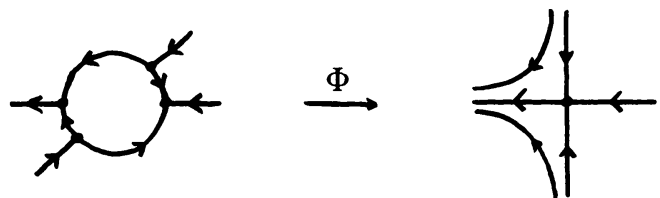

$1 d$.
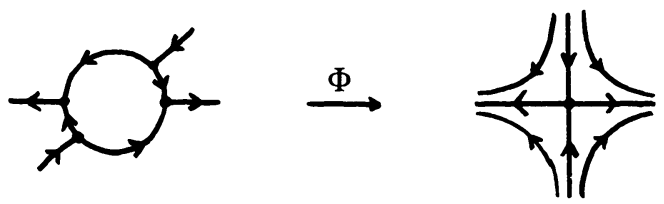

FIgURE 1. Phase portraits of $\bar{X}$ on $S^{1} \times R$ and $X$ on $R^{2}$ 
We now return to the case of potentials that vanish at $(0,0)$ to order $k-1, k \geqslant 2$.

In general let $X$ be an at least $C^{k+1}$ vectorfield (not necessarily a gradient) with its $k$-jet the first nonvanishing jet at $(0,0)$. Let $X_{k}$ denote the vectorfield whose components are homogeneous polynomials of degree $k$ and which has the same $k$-jet as $(0,0)$ as $X$. Let

$$
f=\left\langle x \partial / \partial x+y \partial / \partial y, X_{k}\right\rangle \text { and } g=\left\langle x \partial / \partial y-y \partial / \partial x, X_{k}\right\rangle,
$$

which are homogeneous polynomials of degree $k+1$. The following proposition, a slight extension of the original appearing [7], is implied by the work of Dumortier [2]; it also follows from results in [6, $\$ 4(C)]$.

(2.3) Proposition (CF. [7, P. 66]). Let $X, X_{k}, f$, and $g$ be as above. If $g \neq 0$ but has a line of zeros and if $f(p)=g(p)=0$ implies $p=(0,0)$, then each $C^{k+1}$ vectorfield $Y$ with the same $k$-jet at $(0,0)$ as $X$ is topologically equivalent to $X$ in a nbd of $(0,0)$. In particular $X_{k}$ is topologically equivalent to $X$.

As noted in [7], if all critical points of $\bar{X}$ on $S^{1} \times\{0\}$ are hyperbolic (as when $d g(p)=0$ implies $p=(0,0)$, the hypothesis in the original proposition), then for $Y$ a vectorfield with $(k-1)$-jet zero and $k$-jet at $(0,0)$ near that of $X, Y$ is topologically equivalent to $X$.

By definition, $X_{k}(x, y)=\left[a U_{x}(x, y)+b U(x, y)\right] \partial / \partial x+\left[b U_{x}(x, y)+\right.$ $\left.c U_{y}(x, y)\right] \partial / \partial y$, so equations (2) are

$$
\begin{aligned}
& f(x, y)=(a x+b y) U_{x}(x, y)+(b x+c y) U_{y}(x, y), \\
& g(x, y)=(b x-a y) U_{x}(x, y)+(c x-b y) U_{y}(x, y),
\end{aligned}
$$

or more succinctly,

$$
\left(\begin{array}{l}
f(x, y) \\
g(x, y)
\end{array}\right)=\left(\begin{array}{cc}
x & y \\
-y & x
\end{array}\right)\left(\begin{array}{ll}
a & b \\
b & c
\end{array}\right)\left(\begin{array}{l}
U_{x}(x, y) \\
U_{y}(x, y)
\end{array}\right) .
$$

Since $\left(x^{2}+y^{2}\right)\left(a c-b^{2}\right)=0$ iff $(x, y)=(0,0), f$ and $g$ vanish together precisely where $U_{x}$ and $U_{y}$ do. Since, as is easily checked, the function $g$ in equation (2) has a finite nonzero number of lines of zeros, the following proposition is an immediate consequence of Proposition 2.3.

(2.4) Proposition. For $V=U+W$ as above, if $U_{x}(p)=U_{y}(p)=0$ implies $p=(0,0)$, then $X$ has the same topological type as $X_{k}$. That is, the topological type of $X$ is determined by $U$ and the constant terms of the Riemannian metric.

Note that the condition on $U$ says precisely that it has an isolated singularity at $(0,0)$.

3. Change in type: conditions arising from blowing-up. If at a particular Riemannian metric $g$, with matrix representative $\left(\begin{array}{ll}a & b \\ b & c\end{array}\right)^{-1}$, the singularities on $S^{1} \times\{0\}$ are all hyperbolic, then changing $a, b$, and $c$ slightly moves the $k$-jet of $X$ slightly but keeps the $(k-1)$-jet zero. Thus by the remark following Proposition 2.3 the perturbed vectorfield is equivalent to the original, and the topological type does not change at $g$. The $f$ and the $g$ of the blow-up do not vanish together if $U_{x}$ and $U_{y}$ do not, so that, because 


$$
d \bar{X}\left(\phi_{0}, 0\right)=\left(\begin{array}{cc}
d /\left.d \phi[g(\cos \phi, \sin \phi)]\right|_{\phi=\phi_{0}} & * \\
0 & f\left(\cos \phi_{0}, \sin \phi_{0}\right)
\end{array}\right)=\left(\begin{array}{cc}
\lambda_{1} & * \\
0 & \lambda_{2}
\end{array}\right),
$$

a critical point $p$ of $\bar{X}$ on $S^{1} \times\{0\}$ fails to be hyperbolic iff $d g(p)=0$. These observations lead to the following theorem, stated in the generality allowed by Proposition 2.4.

(3.1) THEOREM. If $V=U+W$ is at least $C^{k+2}$, where $W$ vanishes together with all its partials up to order $k$ at $(0,0)$ and $U(x, y)=\sum_{i=0}^{k} A_{i} x^{k-i} y^{i}$ has no repeated linear factor, then there exists a homogeneous polynomial $P_{k}(x, y)$ of degree $2 k$, with coefficients sums and differences of the $A_{i}$ (of degree 2), $P_{k}$ constructed below, such that the topological type of $X=\operatorname{grad}_{g} V$ can change at some Riemannian metric only if there exists a point $\left(x_{0}, y_{0}\right)$ in $R^{2}$ such that $P_{k}\left(x_{0}, y_{0}\right)>0$.

Proof. By equation (3) and the form of $U(x, y)$ the $g$ of the blow-up is

$$
\begin{aligned}
& g_{x}(0, y)=\left[-2 A_{k-2} a-(k-2) A_{k-1} b+k A_{k} c\right] y^{k-1}, \\
& g_{y}(0, y)=\left[-k A_{k-1} a-k^{2} A_{k} b\right] y^{k-1} .
\end{aligned}
$$

Thus $d g$ vanishes along the line $x=0$ iff

$$
-2 a_{k-2} a-(k-2) A_{k-1} b+k A_{k} c=0, \quad-k A_{k-1} a-k^{2} A_{k} b=0 .
$$

There is a solution to (4) in the cone $\left\{(a, c, b) \mid a, c, a c-b^{2}>0\right\}$ iff

$$
2 k A_{k-2} A_{k}-(k-1)\left(A_{k-1}\right)^{2}>0 \text {. }
$$

That is, there exists a Riemannian metric such that $d g$ vanishes along the line $x=0$ iff inequality (5) holds. Thus $d g$ vanishes along the line $y=m x$ iff in coordinates $(\bar{x}, \bar{y})$ gotten by rotating $y=m x$ to $\bar{x}=0$ we have

$$
2 k \overline{A_{k-2}} \overline{A_{k}}-(k-1)\left(\bar{A}_{k-1}\right)^{2}>0
$$

where $\overline{A_{i}}$ is the coefficient of $\bar{x}^{k-i} \bar{y}^{i}$ for $U$ expressed in $(\bar{x}, \bar{y})$ coordinates. Since $\overline{A_{i}}$ is a homogeneous polynomial of degree $k$ in $u=\cos \alpha$ and $v=\sin \alpha$ (where $\alpha-\pi / 2$ is the angle of rotation of coordinate axes) with coefficients sums and differences of the $A_{i}$, in terms of the original $A_{i}$ the necessary and sufficient condition for $d g$ to vanish along some line, inequality (6), is

$$
\text { there exists }(x, y) \text { such that } P_{k}(x, y)>0 \text {, }
$$

where $P_{k}$ is now as in the statement of the theorem. The theorem now follows from the remarks preceding it.

Although the necessary condition (7) is not in general sufficient for existence of a Riemannian metric at which the topological type of grad $U$ changes, when $\operatorname{deg} U$ $=3$ and, in the presence of hyperbolic sectors, when $\operatorname{deg} U=4$, it is sufficient. In fact, in these cases even more can be said. 
(3.2) Theorem. Let $V=U+W$ be at least $C^{5}$, where $W$ vanishes together with all its partials up to order three at $(0,0)$, and where $U(x, y)=A x^{3}+B x^{2} y+C x y^{2}$ $+D y^{3}$ and the first partials of $U$ vanish together only at $(0,0)$. Then for any Riemannian metric $g, X=\operatorname{grad}_{g} V$ is in one of three topological equivalence classes, as determined by its first nonnvanishing jet (i.e., its 2-jet) at (0, 0): (a) two hyperbolic sectors; (b) two hyperbolic and two parabolic sectors; (c) six hyperbolic sectors. The topological type of $X$ is invariant under changes in the metric iff $X$ has six hyperbolic sectors, which holds iff the resultant $R\left[U_{x}, U_{y}\right]$ is negative, and this latter condition is equivalent to (7).

The resultant $R\left[U_{x}, U_{y}\right]$ is $R\left[U_{x}, U_{y}\right]=3\left(27 A^{2} D^{2}-18 A B C D+4 A C^{3}+\right.$ $\left.4 B^{3} D-B^{2} C^{2}\right)$, and the condition on $U$, that $U_{x}(p)=U_{y}(p)=0$ iff $p=(0,0)$, holds iff the resultant is nonzero. The following lemmas will be used in the proof of the theorem. The straightforward proofs are omitted.

(3.3) LEMMA. The resultant is given by $R\left[U_{x}, U_{y}\right]=L^{2}-4 M N$ where

$$
L=B C-9 A D, \quad M=3 A C-B^{2}, \text { and } N=3 B D-C^{2} \text {. }
$$

The resultant is negative only if both $M$ and $N$ are negative.

Define two new polynomial functions by $g(x, m x)=G(m) x^{3}$ and $f(x, m x)=$ $F(m) x^{3}$. By homogeneity of $g$ and Euler's formula $d g$ vanishing along $y=m_{1} x$ (or $x=0$ ) implies $G$ vanishes with multiplicity at least two at $m_{1}$ (or at infinity).

(3.4) LemMA. Let $U(x, y)=\sum_{i=0}^{k} A_{i} X^{k-y^{i}}$ satisfy $U_{x}(p)=U_{y}(p)=0$ only if $p=(0,0)$. Then there exist admissible perturbations of $a, b$, and $c$ in $\left(\begin{array}{ll}a & b \\ b & c\end{array}\right)$, i.e., so that $a, c, a c-b^{2}>0$ continues to hold, accomplishing the following two perturbations of $G$ : making a zero of even multiplicity disappear and making a zero of odd multiplicity greater than one split into at least three zeros.

The lemma is proved by rotating coordinates to move the root to $m=0$ and using the fact that the conditions on $a, b$ and $c$ are open.

Proof of Theorem 3.2. Suppose that $d g$ does in fact vanish along a line $y=m_{1} x$ for some Riemannian metric. If $G$ vanishes at $m_{1}$ with multiplicity two then $G$ has one other zero $m_{2}$ (which by a small rotation of coordinates may be assumed finite). The condition on $U$ and equation (3) imply $F\left(m_{1}\right) F\left(m_{2}\right) \neq 0$. The condition that $X$ have no elliptic sectors makes only one configuration of signs of $F$ at $m_{1}$ and $m_{2}$ possible, depending on $G$. See Figure 2. By Lemma 3.4 we can by a small change in the metric remove the zero of $G$ at $m_{1}$, i.e., the line of zeros of the $g$ of the blow-up at $y=m_{1} x$, changing the topological type of $X$ by removal of two parabolic sectors (invoking Lemma 1.6). If $G$ vanishes at $m_{1}$ with multiplicity three, it is the sole zero of $G$, and by Lemma 3.4 there is a small change in the Riemannian metric forming two new roots of $G$, i.e., two new lines of zeros of $g$. All three have the same sign under $F$, and the type of $X$ changes by creation of parabolic sectors. Finally, if $d g$ vanishes along the line $x=0$, we may apply the same argument to $g(n y, y)=G(n) y^{3}$ at $n=0$. In sum, the vanishing of $d g$ along a line suffices for change in topological type of $X$. 
<smiles>[3H][C@H]1C[C@@H](C)[C@@H](C)C[C@@H](C)C[C@H]1C</smiles>
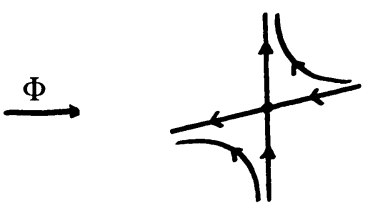

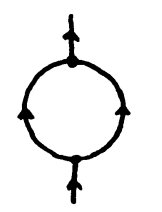
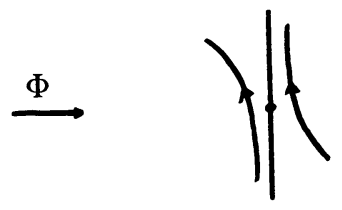
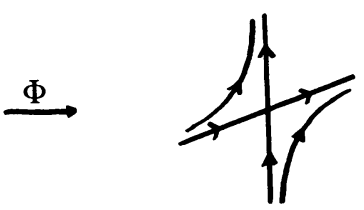<smiles>C[C]1CCCC(I)CC1</smiles>
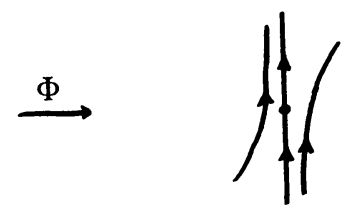

FIGURE 2. Change in topological type of $X$ under perturbation

At this point note that because $f$ takes opposite sign at some pair of adjacent zeros of $g$, which has exactly one, two, or three zeros in all, the phase portrait of $X$ is exactly one of those described in the theorem, up to topological equivalence. Class (c) occurs iff the corresponding zeros of $F$ and $G$ are three in number and alternate along $R$ (plus the point at infinity). Since the zeros of $F$ and $G$ cannot coincide for any Riemannian metric, the topological type is fixed.

Inequality (5) is now $6 B D-2 C^{2}>0$. A direct computation of the new coefficients $\bar{A}, \bar{B}, \bar{C}$, and $\bar{D}$ in terms of the old ones, under a rotation, shows that inequality (6) becomes

$$
\left(M x^{2}+L x y+N y^{2}\right)\left(x^{2}+y^{2}\right)>0
$$

where $L, M$, and $N$ are given by (8). The equivalence of condition (7) and the positivity of the resultant $R\left[U_{x}, U_{y}\right]$ now follows directly from Lemma 3.3.

Finally, by Proposition 1.3, $X$ is in class (a) or (b) iff $U$ has a single line of zeros, which occurs iff $R\left[U_{x}, U_{y}\right]>0$ (provided $R\left[U_{x}, U_{y}\right] \neq 0$ ). But we have just seen that this latter condition holds iff there is a Riemannian metric at which $d g=0$ along a line, which by the first part of the proof holds precisely when the topological type of $X$ can be made to change at that metric. 
Similar techniques may be used to establish the following theorem.

(3.5) TheOREM. Let $V=U+W$ be at least $C^{6}$, where $U$ is a homogeneous polynomial of degree four in $x$ and $y$ whose first partials vanish together only at $(0,0)$ and $W$ vanishes together with all its partials up to order four at $(0,0)$. Then for any Riemannian metric $g, X=\operatorname{grad}_{g} V$ is in one of six topological equivalence classes, as determined by its first nonvanishing jet (i.e., its 3-jet) at zero: (a) sink; (b) source; (c) eight hyperbolic sectors; (d) four hyperbolic and two negative parabolic sectors; (e) four hyperbolic sectors; and (f) four hyperbolic and two positive parabolic sectors. (The case four hyperbolic and four parabolic sectors does not occur.) In the presence of hyperbolic sectors, condition (7) is a necessary and sufficient condition for change in topological type.

It follows from the proof that, as with class (c) of Theorem 3.4, so class (c) here is invariant. From the work of the next section it will follow that $X$ may or may not change from class (e), depending on $U$, while there always exist Riemannian metrics changing $X$ from (d) to (e) and from (f) to (e), but not from (d) to (f) or conversely. It will also follow that all of the equivalence classes are nonempty.

We close this section by pointing out an implication of the proof of the two theorems. For $U$ and $\left(\begin{array}{ll}a & b \\ b & c\end{array}\right)$ as usual, by equation (4) the $g$ of the blow-up of $X=\operatorname{grad}_{g} U$ has derivative $d g$ vanishing along a line $l$ making angle $\alpha$ with the $X$-axis iff

$$
L(\alpha) \cdot\left(\begin{array}{l}
\bar{a} \\
\bar{c} \\
\bar{b}
\end{array}\right)=\left(\begin{array}{c}
-2 \overline{A_{k-2}} \bar{a}-(k-2) \overline{A_{k-1}} \bar{b}+k \overline{A_{k}} \bar{c} \\
-k \overline{A_{k-1}} \bar{a}-k^{2} \overline{A_{k}} \bar{b}
\end{array}\right)=\left(\begin{array}{l}
0 \\
0
\end{array}\right),
$$

where the $\overline{A_{i}}$ and $\bar{a}, \bar{b}, \bar{c}$ are the coefficients of $U$ and the representative of the metric gotten by rotation of coordinate axes through angle $\alpha-\pi / 2$. By homogeneity of $X$ we may identify all metrics whose representatives $(a, c, b)$ lie on the same radial half-line, so distinct metrics correspond to the intersection of the cone $\left\{(a, c, b) \mid a, c, a c-b^{2}>0\right\}$ with $S^{2} \subset R^{3}$. Under the condition on $U, L(\alpha)$ has rank 2; hence $\operatorname{dim} \operatorname{ker}(L)=1$ and the Riemannian metric making $d g$ vanish along $l$ is unique (under the identification) if it exists. If it exists at $\alpha$ it exists at nearby angles, and so we obtain a curve $\gamma(\alpha)=\operatorname{ker} L(\alpha) \cap S^{2}$. In fact the curve exists for all $\alpha$ in $[0,2 \pi)$ and is a closed curve (possibly with self-intersections and even retracings of itself) in $S^{2}$; its intersection with the open cone gives the set of Riemannian metrics at which the topological type of $X$ can (and when $\operatorname{deg} U=3$, does) change.

4. Change in type: geometric conditions. Throughout this section we assume that the hypotheses of Proposition 2.4 hold. Examining the level curves of the potentials $U$ in Theorems $2.2,3.2$, and 3.5 in various cases, one finds that the topological type of the gradient vectorfield $X$ is invariant precisely when level curves of $U$ are concave outward from $(0,0)$ (i.e., outside their tangent lines) at every point. Hence one is led to the conjecture that type can be made to change if and only if such level curves are concave inward at some point.

The necessity is indeed true in general. 
(4.1) TheOREM. If $U$ is a homogeneous polynomial without repeated linear factor and such that level curves $U(x, y) \equiv M \neq 0$ are concave outward from $(0,0)$, then the topological type of $X=\operatorname{grad}_{g} U$ does not change with change in the Riemannian metric $g$.

Proof. By homogeneity of $X_{k}$ its sector-separating null solutions are radial lines, so that the number of parabolic sectors changes exactly when the number of lines along which $X_{k}$ is radially directed, in the region between adjacent lines of zeros of $U$, changes between 1 and $m>1$. Geometrically such lines occur at tangencies of level curves of $U$ with multiples of the unit ball in the norm induced by (the constant terms of) $g$, namely the ellipse $\left(a c-b^{2}\right)^{-1}\left(c x^{2}-2 b x y+a y^{2}\right)=1$, since $X_{k}$ is $g$-perpendicular to level curves of $U$. Thus if level curves are everywhere concave outward from $(0,0)$ between adjacent lines $l_{1}$ and $l_{2}$ of zeros of $U$, then for every family of ellipses there is a unique (line of) such tangency between $l_{1}$ and $l_{2}$, and $X_{k}$ has no parabolic sectors between them (i.e.; between corresponding hyperbolic sectors) for any Riemannian metric.

The sufficiency does not hold in general. In fact, when level curves of $U$ are concave inward along a line in the region between adjacent lines $l_{1}: \theta=\theta_{1}$ and $l_{2}$ : $\theta=\theta_{2}$ of zeros of $U$, there is always a Riemannian metric such that $X_{k}$ has a parabolic sector, i.e., has more than one radial null solution, in the region. The difficulty is that only in low degree are the level curves sure to be so simple that the sector can be "closed."

A technique for counting lines along which $X_{k}$ is radially directed in the region between $l_{1}$ and $l_{2}$ is the following. In the usual polar coordinates $(r, \theta)$, the equation $U \equiv M$ defines $r$ as a smooth function $r=f_{M}(\theta)$ of $\theta$ on $\left(\theta_{1}, \theta_{2}\right)$. By writing the tangent vector to the level curve as a linear combination of $\rho(\theta)=$ $(\cos \theta, \sin \theta)$ and $\tau(\theta)=(-\sin \theta, \cos \theta)$, one has that the tangent is $g$-perpendicular to the radial $\rho(\theta)$, hence $X_{k}$ is radially directed, at values of $\theta \in\left(\theta_{1}, \theta_{2}\right)$ at which

$$
D(\theta)=P(\theta),
$$

where $D(\theta)=-(d / d \theta) \log f_{M}(\theta)$ and

$$
P(\theta)=\frac{R \sin (2 \theta+u)}{K-R \cos (2 \theta+u)},
$$

where $R=\left(\frac{1}{2}(c-a)\right)^{2}+\left[\left(\frac{1}{2}(c-a)\right)^{2}+b^{2}\right]^{1 / 2}, K=\frac{1}{2}(a+c), u$ is the unique number in $[0,2 \pi)$ such that $\frac{1}{2}(c-a)=R \cos u$ and $b=R \sin u$, and $\left(\begin{array}{ll}a & b \\ b & c\end{array}\right)$ is the (constant term) matrix representative of Riemannian metric $g$ (rather than its inverse, as earlier).

$P(\theta)$ of course depends only on $g$, and identifying (by homogeneity) metrics with points of $S^{2}$ inside the open cone $\left\{(a, c, b) \mid a, c, a c-b^{2}>0\right\}$, since $K^{2}-R^{2}=$ $a c-b^{2}$, moving along a curve $u=$ constant in the set of metrics, tending to the curve $a c-b^{2}=0$, makes $K-R$ tend to 0 , and from elementary calculus $P(\theta)$ converges to $C(\theta)=\cot (\theta+u / 2)$ pointwise except at $\theta=-u / 2+n \pi$. On the other hand, $P(\theta)$ shifts to the right or left but is otherwise unchanged as the curve $a c-b^{2}=$ constant is traversed. 
It follows from homogeneity of $U$ and Euler's formula that $D(\theta)=-\cot \alpha(\theta)$, where $\alpha$ is the angle from the radial vector to the tangent. By elementary differential geometry and the fact (arising from the restriction on $U$ ) that the tangent is nowhere collinear with the radial, the curvature $\kappa(\theta)$ of the plane curve $U=M$ satisfies

$$
\begin{gathered}
\kappa>0 \text { when } U \equiv M \text { is concave inwards towards }(0,0), \\
\kappa<0 \text { when } U \equiv M \text { is concave outwards from }(0,0),
\end{gathered}
$$

and

$$
\kappa(\theta)>0 \text { iff } \alpha^{\prime}(\theta)>-1 .
$$

We may now state the result mentioned above.

(4.2) Proposition. Given a level curve $U(x, y) \equiv M>0$ with asymptotes $\theta=\theta_{1}$ and $\theta=\theta_{2}$, there is a Riemannian metric giving $X=\operatorname{grad} U$ more than one radial null solution in $\left(\theta_{1}, \theta_{2}\right)$ iff the level curve is concave inwards toward the origin at some point.

Proof. In the case $\theta_{2}-\theta_{1}=\pi$, it is readily established that $\alpha^{\prime}>-1$ at some point and that (9) has more than one solution for some Riemannian metric. If $\theta_{2}-\theta_{1}<\pi$, it is a simple matter to check that there is a number $u \in[0,2 \pi)$ such that $D(\theta)=C(\theta)=\cot (\theta+u / 2)$ has more than one solution in $\left(\theta_{1}, \theta_{2}\right)$ iff $\alpha^{\prime}\left(\theta_{0}\right)>-1$ for some $\theta_{0} \in\left(\theta_{1}, \theta_{2}\right)$. Thus it suffices to show that (9) has more than one solution in $\left(\theta_{1}, \theta_{2}\right)$, for some choice of $a, b$, and $c$, hence of $u$, iff $D(\theta)=C(\theta)$ does. Sufficiency is clear from convergence of $P$ to $C$ for appropriate choice of metric. Conversely, if for some choice of metric $D$ meets $P$ twice, the case of interest is $D^{\prime}(\theta)<0$ on $\left(\theta_{1}, \theta_{2}\right)$, and then we may shift $P$ so that at some $\theta_{0} \in\left(\theta_{1}, \theta_{2}\right)$, $D\left(\theta_{0}\right)=P\left(\theta_{0}\right)$ and $D^{\prime}\left(\theta_{0}\right)=P^{\prime}\left(\theta_{0}\right)<0$. But then a calculus computation yields $P^{\prime}\left(\theta_{0}\right)>C^{\prime}\left(C^{-1}\left(P\left(\theta_{0}\right)\right)\right)$; hence a second shift in the amount $C^{-1}\left(P\left(\theta_{0}\right)\right)-\theta_{0}$ causes $D\left(\theta_{0}\right)=C\left(\theta_{0}\right)$ but $D^{\prime}\left(\theta_{0}\right)>C^{\prime}\left(\theta_{0}\right)$. Since $\theta_{2}-\theta_{1}<\pi=$ period $C$, an examination of the sign of $D-C$ near the asymptotes of $C$ leads to the existence of a second intersection.

Proposition 4.2 in fact implies that there will be topological change in the phase portrait of $X$ between adjacent hyperbolic sectors, corresponding to adjacent half-lines of zeros of the potential function, iff level curves in the region are concave inward towards the origin at some point and there exists a Riemannian metric giving $X$ a unique null solution in the region. The question then is when the latter condition holds. The following lemma, which will be interpreted geometrically below, gives a partial answer.

(4.3) LEMMA. If $D(\theta)$ can be placed (by rotation of coordinate axes) so as not to intersect $\cot \theta$ (equivalently, $u$ can be chosen so that $C(\theta)$ does not intersect $D(\theta)$ ), then there is a Riemannian metric giving a unique solution to (9) on $\left(\theta_{1}, \theta_{2}\right)$. If $D(\theta)$ can be placed in $[-\pi / 2, \pi / 2]$ (equivalently, $u$ chosen as above) so as to intersect $\cot \theta$ at $\theta_{3}<0<\theta_{4}$, and if $-\cot \theta_{4} \leqslant D(0) \leqslant-\cot \left(\pi+\theta_{3}\right)$, then there is more than one solution to (9) in $\left(\theta_{1}, \theta_{2}\right)$ for every Riemannian metric. 
Proof. The proof of the first statement is obvious, given the control that we have over $P$ by changing the Riemannian metric.

Now suppose that $D(\theta)$ is placed in $[-\pi / 2, \pi / 2]$ so as to intersect $\cot \theta$ at $\theta_{3}, \theta_{4}$, $\theta_{3}<0<\theta_{4}$. If $D(0) \neq 0$ it may be possible to shift $D$ slightly and obtain a curve $P(\theta)$ that meets $D(\theta)$ only once. If however $D(0)=0$ originally, it is easily checked that however $D$ (or $u$ ) is shifted $D$ and $P$ always meet more than once. The inequality condition will be justified below.

If $D\left(\theta_{i}\right)=\cot \left(-\alpha\left(\theta_{i}\right)\right)=\cot \theta_{i}$ for $i=3,4$, where $-\pi<\theta_{3}<0<\theta_{4}<\pi$, then $-\pi<-\alpha(\theta)<0$ implies $\alpha\left(\theta_{3}\right)=-\theta_{3}$ and $\alpha\left(\theta_{4}\right)=\pi-\theta_{4}$. Geometrically this means that the tangents to $U(x, y) \equiv M$ are horizontal along $\theta=\theta_{3}$ and $\theta=\theta_{4}$ and oppositely directed. The quantity $D(0)$ expresses the angle the level curve makes with the $X$-axis, and is zero iff this angle is $\pi / 2$. If $D(0) \neq 0$ there is an invertible linear change of coordinates preserving the $X$-axis and making $D(\overline{0})=0$ (new coordinates). The horizontal tangents continue horizontal, and if the original tangent at the $X$-axis was sufficiently close to vertical (the condition on $D(0)$ ) then in the new coordinates $-\pi / 2 \leqslant \bar{\theta}_{3}<0<\bar{\theta}_{4} \leqslant \pi / 2$ so the discussion in the case $D(0)$ zero applies to show that there are at least two null solutions of $X$.

Thus Lemma 4.3 says that if level curves do not have pairs of oppositely directed tangents parallel to a radial line separating them, positive curvature of level curves suffices for change in a parabolic sector. It also shows that presence of such tangents can but need not obstruct closing of a parabolic sector.

When the level curves between all lines of zeros of $U$ are considered together, a second obstruction to the sufficiency of the concavity condition might occur, namely, parabolic sectors might be "linked" in such a way that one pair closes iff another opens, leaving the topological type of the vectorfield unchanged. However, this requires $k=\operatorname{deg} U$ at least seven and could only occur on the complement of a dense open set of homogeneous potential functions of fixed degree.

The following theorem, which has an obvious generalization in view of Proposition 2.4, summarizes the results of this section. Define:

Condition 1. Some level curve of $U$ is concave inwards toward the origin at some point (i.e., $\kappa>0$ at some point).

Condition 2. No radial line separates and is parallel to two oppositely directed tangents on a level curve of $U$.

(4.4) TheOREM. Let $U$ be a homogeneous polynomial of degree $k$ and such that $U_{x}(p)=U_{y}(p)=0$ implies $p=(0,0)$. Then

(a) Condition 1 is a necessary condition for the topological type of $X=\operatorname{grad}_{g} U$ to change at some (constant) Riemannian metric $g$.

(b) For $k \leqslant 4$ (and $k=5$ when $U$ has more than one line of zeros) condition 1 and existence of a line of zeros of $U$ is a necessary and sufficient condition for change.

(c) For an open and dense set of homogeneous polynomials of degree $k$, conditions 1 and 2 are sufficient for change.

Proof. Part (a) is Proposition 4.2. In part (b), the cases $k=2$ and $k=3$ follow from Theorems 2.2 and 3.2 respectively. Hence suppose $k=4$. Then the condition 
on $U$ implies that it has two or four lines of zeros and changes sign at each. If there are four, level curves are concave outward everywhere. Hence suppose $U$ has lines of zeros $l_{1}: \theta=\theta_{1}$ and $l_{2}: \theta=\theta_{2}, 0<\theta_{1}<\theta_{2}<\pi$, and $U(x, 0)>0$. Differentiating the equation $U(x, y) \equiv M$ implicitly with respect to $x$ and using the condition on $U(x, y)$ yields $d y / d x=0$ iff $U_{x}=0 . U_{x}$ is positive (resp. negative) on $l_{1}$ (resp. $l_{2}$ ) when $y>0$, the opposite sign when $y<0$; it therefore has an odd number of lines of zeros in $\theta_{1}<\theta<\theta_{2}$. As a cubic it can have at most two lines of zeros in $\theta_{2}-\pi<\theta<\theta_{1}$, and must change sign at each. Thus they cannot be of the form $l_{3}$ : $\theta=\theta_{3}<0, l_{4}: \theta=\theta_{4}>0$, else $U_{x}$ would be negative on the positive $X$-axis, which is impossible since $U(0,0)=0$. $U$ cannot then have oppositely directed tangents parallel to the $X$-axis and above and below it, hence cannot have such tangents parallel to a radial line, which could be rotated to the $X$-axis. Lemma 4.3 now implies the sufficiency of condition 1 for change in type. The proof for the case $k=5$ when $U$ has three lines of zeros is similar.

For part (c), by Lemma 4.6 we need only consider the sort of "linkage" of parabolic sectors mentioned above. By a long but straightforward computation, keeping track of the zeros of the $g$ of the blow-up and adjusting $\left(\begin{array}{ll}a & b \\ b & c\end{array}\right)$ finitely many times, it follows that if linkage of parabolic sectors occurs for $U$ at Riemannian metric $g$, then there is $U^{\prime}$ arbitrarily close to $U$ and $g^{\prime}$ close to $g$ such that the topological type of $X^{\prime}$ changes at $g^{\prime}$.

As a final note we remark that this theorem explains why, as mentioned earlier, in $\operatorname{deg} U=4$ there always exist Riemannian metrics changing $X$ from classes (d) and (f) in Theorem 3.5 to class (e), but (d) can never change to (f) or conversely.

\section{REFERENCES}

1. A. A. Andronov et al., Qualitative theory of second order dynamic systems, John Wiley \& Sons, New York, 1973.

2. F. Dumortier, Singularities of vectorfields in the plane, J. Differential Equations 23 (1977), 53-106.

3. John Guckenheimer, Bifurcation and catastrophe in dynamical systems (M. M. Peixoto, ed.), Academic Press, New York, 1973.

4. Philip Hartman, Ordinary differential equations, John Wiley \& Sons, New York, 1964.

5. Nicolaas Kuiper (Editor), Manifolds-Amsterdam 1970, Lecture Notes in Math., vol. 197, SpringerVerlag, New York, 1971.

6. J. Palis and F. Takens, Topological equivalence of normally hyperbolic dynamical systems, Topology 16 (1977), 335-345.

7. F. Takens, Singularities of vectorfields, Inst. Hautes Études Sci. Publ. Math. 43 (1974), 47-100.

Department of Mathematics, University of North Carolina at Charlotte, Charlotte, North Carolina 28223 